\title{
Correlation Between the Severity of Coronary Artery Ectasia and Monocyte/Lymphocyte, Platelet/Lymphocyte, and HDL/LDL Ratios
}

\author{
Emre Özdemir1, Özgen S,afak², Mert Pehlivan Altın1, Didar Elif Akgün², Sadık Volkan Emren¹, Eyüp Avcl², \\ Mehmet Tokaç1 and Filiz Akçay Akyıldız' \\ ${ }^{1}$ Cardiology Clinic, Izmir Katip Çelebi University, Ataturk Education and Research Hospital, Izmir, Turkey \\ ${ }^{2}$ Cardiology Clinic, Balikesir University, Education and Research Hospital, Balikesir, Turkey
}

\begin{abstract}
Objective: To evaluate the efficacy of the monocyte/lymphocyte, platelet/lymphocyte, and HDL/LDL ratios as markers of the severity of coronary artery ectasia(CE).

Study Design: Cross-sectional study.

Place and Duration of Study: Department of Cardiology, Izmir Katip Celebi University and Balikesir University, Turkey, from January 2017 to October 2018.

Methodology: A total of 7,923 coronary angiographs were retrospectively scanned. Inclusion criteria was $>1.5$ times dilatated of native coronary artery segment. Exclusion criteria was $<1.5$ times or no dilatation of native coronary segment compared with normal coronary segment. Demographic features, CE type, clinic status, monocyte/lymphocyte, platelet/ lymphocyte and HDL/LDL ratios are collected.

Results: Two hundred and six (2.6\%) cases were identified, which had a mean age of $61.4 \pm 11.4$ years. The male to female ratio was $3: 1$; and $46 \%$ of the patients presented with unstable angina. The prevalence of CE was $2.6 \%$; and 118 $(57.2 \%)$ patients had non-obstructive coronary artery disease. Hypertension, hyperlipidemia and smoking were the most commonly seen disorders. Markis Type 4 was the most common type of CE determined. A statistically significant correlation was determined among the monocyte/lymphocyte ratio, platelet/lymphocyte ratio, and the increased diameter of ectasia of the vessel.

Conclusion: Although there should be awareness of $\mathrm{CE}$, many clinicians do not pay as much attention to $\mathrm{CE}$ as to coronary stenosis. The results of this study showed that the monocyte/lymphocyte ratio and platelet/lymphocyte ratio are consistent with the ectasia severity. This correlation will be useful for the evaluation of follow-up and treatment success in patients with CE.
\end{abstract}

Key Words: Coronary artery ectasia, High-density lipoprotein/low-density lipoprotein ratio, Lipoprotein ratio, Monocyte/lymphocyte ratio, Platelet/lymphocyte ratio.

How to cite this article: Özdemir E, Şafak Ö, Altın MP, Akgün DE, Emren SV, Avcl E, Tokaç M, Akylldı FA. Correlation between the severity of coronary artery ectasia and monocyte/lymphocyte, platelet/lymphocyte, and HDL/LDL ratios. J Coll Physicians Surg Pak 2020; 30(3):235-239.

\section{INTRODUCTION}

Coronary artery ectasia is defined as dilatation of $>1.5$ times the normal coronary artery segment, and focal coronary dilatation is known as coronary aneurysm (Figure 1). ${ }^{1}$ Coronary ectasia has a reported prevalence of $0.3 \%-4.9 \%$ and male dominance at a ratio of $3: 1.2$

Markis et al. classified this pathology into four groups. ${ }^{3}$ Type 1 is defined when there is diffuse ectasia in two or three vessels. Type 2 is labelled when there is diffuse ectasia in a vessel and localised disease (aneurysm) in another vessel. Type 3 is defined when there is diffuse ectasia in only one vessel, and Type 4 is defined when

Correspondence to: Dr. Emre Özdemir, Cardiology Clinic,

Katip Celebi University, Ataturk Education and Research Hospital,

Izmir, Postal Code 35620, Turkey

E-mail: emreozdemir27@yahoo.com.tr

Received: September 17, 2019; Revised: January 15, 2020;

Accepted: February 12, 2020 there is a localised or segmental disease (aneurysm) in only one vessel.

Coronary ectasia may be asymptomatic or present as a clinic of myocardial ischemic symptoms or acute coronary syndromes. ${ }^{4}$ Coronary ectasia may occur with a different reflection of atherosclerosis or with some inflammatory events such as enzymatic corruption, atherosclerotic process of the extracellular matrix. ${ }^{5}$

But many clinicians do not pay as much attention to coronary ectasia as coronary stenosis. They should be aware of coronary artery ectasia. There are no definitive treatment options and different studies have shown different risk scales. ${ }^{6}$

Despite all this information and studies related to coronary ectasia, this pathology is still an interesting and unknown disease. ${ }^{7}$

The aim of this study was to provide information about the classification and follow-up of coronary artery 
ectasia, a disease that tends to be overlooked by clinicians; and to identify a marker of coronary artery ectasia severity. The hypothesis of the study was that the monocyte/lymphocyte ratio and the platelet/ lymphocyte ratio may be effective in the follow-up and evaluation of treatment for coronary artery ectasia.

\section{METHODOLOGY}

A retrospective review was made of the angiography examinations from two different departments - Department of Cardiology, Izmir Katip Celebi University and Balikesir University, Turkey - both referral tertiary health centres, between January 2017 and October 2018. From a total of 7,923 coronary angiographs, 206 (2.6\%) were identified with coronary artery ectasia.

The ectasias and aneurysms noted were determined according to the criteria in literature. Coronary ectasia was defined as $>1.5$-fold greater dilatation from the normal coronary segment, and aneurysm was defined as discrete dilatation. Inclusion criteria was $>1.5$ times dilatated native coronary artery segment. Exclusion criteria was defined as $<1.5$ times or no dilatation on native coronary segment compared with normal coronary segment. All ectasias were classified according to the Markis definition 3 as defined before. Coronary ectasia segment locations were labelled (Right coronary artery (RCA), circumflex artery (Cx), and left anterior desending artery (LAD)).

Comorbid diseases included hypertension, diabetes mellitus, dyslipidemia, and chronic obstructive lung disease, which were respectively defined as arterial blood pressure regulated by at least one drug or diet, blood glucose or total cholesterol, and inhaler drug use for lung disease. Chronic kidney disease was defined as a low glomerular filtration rate for age. Patient administration clinic (unstable/stable angina or nonST/ST elevated myocardial infarction), lymphocyte, leukocyte, monocyte, hemoglobin, platelet, platelet volume, total cholesterol, triglyceride, high density lipoprotein levels (HDL) and low density lipoprotein (LDL), high density lipoprotein/low density lipoprotein ratiomonocyte/lymphocyte, platelet/lymphocyte, and HDL/LDL ratios were recorded. All data were collected retrospectively from the hospital records of the two centres. Ethics Committee approval received from local Ethics Committee of Izmir Katip Çelebi University, Ataturk Education and Reserch Hospital prior to initiation of the research work.

Data obtained in the study were analysed statistically using SPSS for Windows version 11.5 software (SPSS Inc. Co., Chicago, IL, USA). Conformity to normal distribution of continuous variables was tested using the Kolmogorov-Smirnov test. Descriptive data were expressed as mean \pm standard deviation (SD) values for continuous variables with normal distribution and as median (min-max) values for those with non-normal distribution. The relationships between vascular diameter, monocyte/lymphocyte ratio, platelet/lymphocyte ratio, and high density lipoprotein/low density lipoprotein ratio were evaluated using Spearman's RHO analysis.

\section{RESULTS}

Retrospective evaluation was made of a total of 7,923 coronary angiographs taken between January 2017 and October 2018. All coronary ectasias and aneurysms were identifed and the Markis classification was noted. ${ }^{3}$ Comorbid diseases were recorded as hypertension, hyperlipidemia, diabetes mellitus, chronic obstructive pulmonary diseases, and chronic kidney disease. Coronary artery ectasia was detected in 206 (2.6\%) cases, nearly half of which had been overlooked and not recorded as coronary ectasia on the angiography report. There was male gender dominance with 158 (76\%) male patients, and mean age was $62 \pm 8$ years for males and $57 \pm 9$ years for females. The most prevalent clinical presentation was unstable angina pectoris at the rate of $44.6 \%$ (n:92) followed by stable angina pectoris at $31.3 \%$ (n:64), non-ST elevated myocardial infarction at $17.4 \%$ (n:36), and ST elevated myocardial infarction at $6.7 \%(\mathrm{n}: 14)$.

Hypertension was the most frequent comorbid disease at the rate of $66 \%$ ( $\mathrm{n:168)}$, followed by hyperlipidemia at $62 \%(\mathrm{n}: 136)$, smoking at $60 \%(\mathrm{n}: 123)$, and diabetes mellitus at $38 \%(\mathrm{n}: 76)$. Chronic obstructive pulmonary disease and chronic kidney disease were seen less frequently.

According to the Markis classification 3, Type 4 coronary ectasia was most frequent at $55.3 \%$ ( $\mathrm{n}: 57)$, followed by Type 2 at $22.4 \%(n: 23)$, Type 1 at $11.7 \%(n: 12)$, and Type3 at $10.6 \%(\mathrm{n}: 11)$.

According to the diameter of the ectasia/aneurysm, $42.8 \%(\mathrm{n}: 88)$ of the coronary artery ectasias were $<5 \mathrm{~mm}$, $53.6 \%(\mathrm{n}: 111)$ were $5-8 \mathrm{~mm}$, and $3.6 \%(\mathrm{n}: 7)$ were $>8 \mathrm{~mm}$.

Coronary ectasia was most frequently detected in the right coronary artery (RCA) at the rate of $59.2 \%$ ( $\mathrm{n}: 122)$, followed by the left anterior desending artery (LAD) at $42.7 \%(n: 88)$, the circumflex artery (Cx) at $40.7 \%(n: 84)$, and the left main coronary artery(LMCA) at $0.9 \%$ (n:2).

Mean lymphocyte (LYM) count was determined as 2.12 $\pm 0.8 \times 109 / \mathrm{L}$, mean leukocyte (LEU) count as 8.95 $\pm 3.1 \times 109 / \mathrm{L}$, and mean monocyte (MON) count as 0.75 $\pm 2.3 \times 109 / \mathrm{L}$. The mean hemoglobin $(\mathrm{Hb})$ level was 13.2 $\pm 2.0 \mathrm{~g} / \mathrm{L}$, mean platelet (PLT) count was $245.8 \pm 87.5$ x109/L, mean platelet volume (MPV) was $10.2 \pm 1.5 \mathrm{fL}$, mean total cholesterol (TC) was $189.2 \pm 47.5 \mathrm{mg} / \mathrm{dL}$, mean triglyceride (TG) was $167 \pm 89.0 \mathrm{mg} / \mathrm{dL}$, mean high density lipoprotein (HDL) was $19.7 \pm 9.4 \mathrm{mg} / \mathrm{dL}$, and mean low density lipoprotein (LDL) was $118.4 \pm 38.2$ $\mathrm{mg} / \mathrm{dl}$. The mean MON/LYM ratio was calculated as 


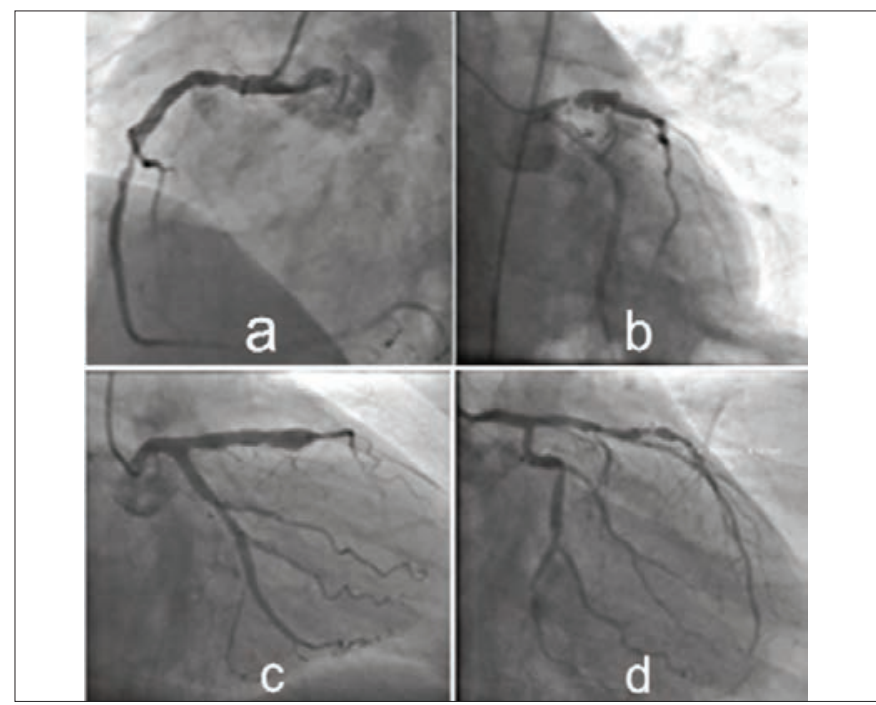

Figure 1: (a) proximal segment ectasia of RCA; (b) In-stent proximal segment ectasia of LAD with severe coronary stenosis; (c) Native LAD diffuse aneurysm; (d) multiple ectasias on $\mathrm{Cx}$.

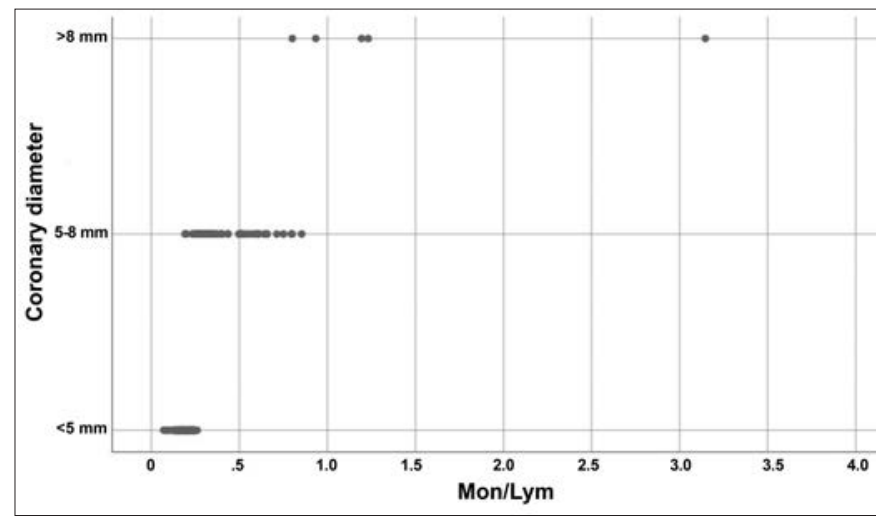

Figure 2: Monocyte / lymphocyte ratio and coronary ectasia diameter distribution.

$0.34 \pm 0.30$, mean PLT/LYM ratio as $142.3 \pm 82.9$, and mean HDL/LDL ratio as $0.38 \pm 0.2$.

A statistically significant relationship was determined between the MON/LYM ratio $(r=0.843, p<0.001)$, PLT/LYM ratio $(r=0.475, p<0.001)$ and increased diameter of coronary artery ectasia. When this ratio was low, the prevalence of $<5 \mathrm{~mm}$ ectasia was low but when the MON/LYM ratio increased then the prevalence of $>5 \mathrm{~mm}$ ectasia increased. No correlation was determined between the HDL/LDL ratio and ectasia diameter $(r=-0.031, p=0.733)$ (Figure 2).

\section{DISCUSSION}

Coronary ectasia is associated with arteriosclerosis in adults and Kawasaki disease in children.8 Coronary artery revascularisation procedures, such as balloon angioplasty or stent implantation, congenital malformations, chest traumas, connective tissue disorders such as Behcet's disease, and collagenopathies such as Marfan syndrome may play a role in the development of coronary artery ectasia. 5
The pathological process is associated with enzymatic degradation of the extracellular matrix by metalloproteinase, cysteine proteinases, and serine proteinases. 9 This degradation and vessel dilatation leads to a deterioration in laminar coronary blood flow. The clinical table of coronary artery ectasia patients is associated with concomitant CAD severity and deterioration of laminar coronary flow. Dilatation severity is associated with coronary blood flow quality. These two conditions should be considered together; and the treatment should be managed accordingly. 10

In the current study cohort, evaluation was made of 7,923 coronary angiographic records; and 206 cases of coronary artery ectasia were identified, at a prevalence rate of $2.6 \%$. This was seen to be similar to previously reported coronary artery ectasia prevalence data. ${ }^{2}$

The mean age for the whole cohort was $61.4 \pm 11.4$ years, which was also similar to the findings of Aksu et al. 11 The male predominance $(76 \%, \mathrm{n}: 158)$, of the current study was compatible with the data of a previous Turkish study by Aksu et al.,11 and similar to that of Qin et al. 12

In a coronary artery ectasia review, Devabhaktuni et al. reported that hypertension and smoking are the most common risk factors; 13 whereas, diabetes mellitus is a low risk factor for coronary artery ectasia. This paradox may be explained by the decrease of proteinase activity with negative remodelling to atherosclerosis in diabetes treatment. ${ }^{14,15}$ That hypertension was the most common comorbid disease of this study and was compatible with these data; and diabetes was not seen as frequently as hyperlipidemia and hypertension.

Aboeata et al..$^{16}$ reported that stable angina pectoris is the most common presentation for coronary artery ectasia patients. ${ }^{16}$ Decreased coronary flow and atherosclerosis formation can also result from acute coronary syndromes such as unstable angina pectoris. 17 In the current study, unstable angina pectoris was the most common clinical presentation ( $\mathrm{n}: 92,44.6 \%)$, followed by stable angina pectoris $(\mathrm{n}: 64,31.3 \%)$. Non-ST elevated myocardial infarction ( $\mathrm{n}: 36,17.4 \%)$ and ST elevated myocardial infarction ( $\mathrm{n}: 14,6.7 \%$ ) was detected more rarely. In the light of this information, it can be concluded that the clinical reflection is due to slow coronary flow rather than atherosclerosis.

Qin et al. and Aksu et al. reported that coronary artery ectasia arterial involvement is most frequently seen in the RCA, then $C x$ and $L A D$, and less frequently in the LMCA. ${ }^{11,12}$ Similarly, in the current study, RCA involvement was most common, determined at the rate of $59.2 \%$ ( $n: 122)$, and LAD and Cx were seen at similar rates of $42.7 \%(n: 88)$ and $40.7 \%(n: 84)$, respectively. LMCA involvement was much less frequent at $0.9 \%$ $(n: 2)$. 
According to the Markis classification, Type 4 coronary artery ectasia was the most common ectasia type in this study, which was compatible with literature. It has also been reported that Type 1 and Type 2 coronary artery ectasia have a worse prognosis than Types 3 and 4.3,11

Chen et al. reported that the mon/lym ratio is associated with the severity of coronary atherosclerotic disease and is a predictor for major adverse cardiac events such as non-ST elevated myocardial infarction. ${ }^{18}$ Sudhir et al. and Saigusa et al. demonstrated a correlation between coronary artery ectasia and serum lipid levels. ${ }^{19,20}$ Qin et al. also detected a higher LDL/HDL ratio in coronary artery ectasia patients. 12 However, Yasar et al. and Moghadam et al. reported a correlation between platelet activity and coronary artery ectasia. 21,22

In the light of the available information, it is possible to presume a relationship between coronary ectasia and various laboratory parameters. These interactions may be related to both inflammatory and thrombotic system activation.

Consistent with previous studies, the results of the current study also showed that high MON/LYM and PLT/LYM ratios are associated with coronary artery ectasia vessel diameter, and no correlation was determined between the HDL/LDL ratio and ectatic vessel diameter.

There is no definitive treatment for coronary artery ectasia because of the variety of patient profiles. Furthermore, medical treatment of coronary artery ectasia may include acetylsalicylic acid, high dose statins and anti-ischemic medications (calcium channel blocker, etc), and atherosclerotic risk factor modification is required. 10,23

This modification and medication should be implemented considering the stenosis and ectasia status of coronary artery disease together. According to the current study results, there was a statistically significant relationship between the MON/LYM and PLT/LYM ratios and the severity of coronary artery ectasia. These ratios can be useful for follow-up of the patient.

\section{CONCLUSION}

Several factors have been associated with coronary ectasia, including lipoprotein concentration, the inflammatory milieu, renin-angiotensin system, homocysteine levels, insulin, nitric oxide, and atherosclerosis. In the current study, a correlation was determined between the MON/LYM and PLT/LYM ratios and ectasia diameter. This correlation will be useful in the evaluation of changes during the follow-up of ectasia severity and treatment success in patients with coronary artery ectasia. It may also be considered that this may prevent recurrent coronary angiography.

\section{ETHICAL APPROVAL:}

Ethics Committee approval was received for this study from the local Ethics Commitee (2019-386).

\section{PATIENTS' CONSENT:}

As the study was designed retrospectively, data was collected from clinical archive after ethical approval.

\section{CONFLICT OF INTEREST:}

Authors declared no conflict of interest.

\section{AUTHORS' CONTRIBUTION:}

EÖ: Study design, data collection, literature review and writing.

ÖS: Drafting of work.

SVE: Statistical analysis.

MPA, DEA: Data collection.

EA, FAA, MT: Final review and approval of article.

\section{REFERENCES}

1. Swaye PS, Fisher LD, Litwon P, Vingela PA, Judkins MP, Kemp $\mathrm{HG}$, et al. Aneurysmal coronary artery disease. Circulation 1983; 67:134-8.

2. Hartnell GG, Parnell BM, Pridie RB. Coronary artery ectasia. Its prevalence and clinical significance in 4993 patients. $\mathrm{Br}$ Heart J 1985; 54:392-5.

3. Markis JE, Joffe CD, Cohn PF, Feen DJ, Herman MV, Gorlin R. Clinical significance of coronary arterial ectasia. $A m \mathrm{~J}$ Cardiol 1976; 37:217-22.

4. Rashid S, Gul U, Ali M, Sadiq T, Kiyani AM. Coronary artery ectasia: Clinical and angiographic features. J Coll Physicians Surg Pak 2018; 28:824-8.

5. Antoniadis AP, Chatzizisis YS, Giannoglou GD. Pathogenetic mechanisms of coronary ectasia. Int J Cardiol 2008; 130:33543.

6. Ibarra-Torres A, Cabrera-Leal C, López-Medina G, Soto ME, Vallejo E, Rodríguez-Galván A, et al. Giant aneurysm of the right coronary artery, report of a case and review of the literature. Arch Cardiol Mex 2019; 89:248-53.

7. Boles U. Insight into coronary artery ectasia. PhD Thesis. Umea University 2019.

8. Zeina AR, Sharif $D$, Blinder J, Rosenschein U, Barmeir E. Non-invasive assessment of coronary artery ectasia using multi-detector computed tomography. Coron Artery Dis 2007; 18: $175-80$.

9. Liu J, Sukhova GK, Yang JT, Sun J, Ma L, Ren A, et al. Cathepsin $\mathrm{L}$ expression and regulation in human abdominal aortic aneurysm, atherosclerosis, and vascular cells. Atherosclerosis 2006; 184:302-11.

10. Mavrogeni S. Coronary artery ectasia: From diagnosis to treatment. Hellenic J Cardiol 2010; 51:158-63.

11. Aksu T, Uygur B, Kosar MD, Guray U, Arat N, Korkmaz S, et al. Coronary artery ectasia: Its frequency and relationship with atherosclerotic risk factors in patients undergoing cardiac catheterization. Anatol J Cardiol 2011; 11:280-4.

12. Qin Y, Tang C, Ma C, Yan G. Risk factors for coronary artery ectasia and the relationship between hyperlipidemia and coronary artery ectasia. Coron Artery Dis 2019; 30:211-5.

13. Devabhaktuni S, Mercedes A, Diep J, Ahsan C. Coronary 
artery ectasia: A review of current literature. Curr Cardiol Rev 2016; 12:318-23.

14. Baugh MD, Gavrilovic J, Davies IR, Hughes DA, Sampson MJ. Monocyte matrix metalloproteinase production in Type 2 diabetes and controls: A cross sectional study. Cardiovasc Diabetol 2003; 2:3.

15. Kornowski R, Mintz GS, Lansky AJ, Hong MK, Kent K, Pichard $A D$, et al. Paradoxic decreases in atherosclerotic plaque mass in insulin-treated diabetic patients. Am J Cardiol 1998; 81:1298-304.

16. Aboeata AS, Sontineni SP, Alla VM, Esterbrooks DJ. Coronary artery ectasia: Current concepts and interventions. Front Biosci Elite Ed 2012; 4:300-10.

17. Ozcan OU, Gulec S. Coronary artery ectasia. Cor et Vasa 2013; 55:e242-7.

18. Chen H, Li M, Liu L, Dang X, Zhu D, Tian G. Monocyte/ lympho-cyte ratio is related to the severity of coronary artery disease and clinical outcome in patients with non-ST-elevation myo-cardial infarction. Medicine 2019; 98:e162-7.
19. Sudhir K, Ports TA, Amidon TM, Goldberger JJ, Bhushan V, Kane JP, et al. Increased prevalence of coronary ectasia in heterozygous familial hypercholesterolemia. Circulation 1995; 91:1375-80.

20. Saigusa T, Izawa A, Miura T, Ebisawa S, Shiba Y, Miyashita Y, et al. Low levels of high-density lipoprotein cholesterol predict the presence of coronary artery disease in patients with aortic aneurysms. Angiology 2014; 65:710-5.

21. Yasar AS, Erbay AR, Ayaz S, Turhan H, Metin F, Ilkaya E, et al. Increased platelet activity in patients with isolated coronary artery ectasia. Coron Artery Dis 2007; 18:451-5.

22. Moghadam RH, Shahmohammadi A, Asgari N, Azizi K, Mansour SM, Roozbahani M. Comparison of mean platelet volume levels in coronary artery ectasia and healthy people: Systematic review and meta-analysis. Blood Res 2018; 53: 269-75.

23. Turhan $H$, Yetkin $E$. What is the plausible strategy for the management of patients with isolated coronary artery ectasia and myocardial ischemia. Int J Cardiol 2007; 117:285-6. 